\title{
Écriture de la ville et poétique du mensonge - l'espace urbain chez Flaubert et Balzac
}

Stadtliteratur und Poetik der Lüge - der urbane Raum im Werk Flauberts und Balzacs

Writing the city and poetics of the lie - urban space in Flaubert's and Balzac's novels

Christine Schmider

\section{OpenEdition}

\section{Journals}

Édition électronique

URL : http://journals.openedition.org/ceg/1405

DOI : 10.4000/ceg. 1405

ISSN : 2605-8359

\section{Éditeur}

Presses Universitaires de Provence

\section{Édition imprimée}

Date de publication : 15 juin 2015

Pagination : 49-61

ISBN : 978-2-85399-993-9

ISSN : 0751-4239

\section{Référence électronique}

Christine Schmider, «Écriture de la ville et poétique du mensonge - l'espace urbain chez Flaubert et Balzac », Cahiers d'Études Germaniques [En ligne], 68 | 2015, mis en ligne le 17 décembre 2017, consulté le 01 décembre 2020. URL : http://journals.openedition.org/ceg/1405 ; DOI : https://doi.org/ $10.4000 /$ ceg. 1405 


\section{Écriture de la ville et poétique du mensonge - l'espace urbain chez Flaubert et Balzac \\ Christine SCHMIDER \\ Université Nice - Sophia Antipolis}

« Plus encore qu'un sujet, le lieu d'une fiction peut être sa vérité ${ }^{1}$ » constate Roland Barthes dans Le Degré zéro de l'écriture. Nous nous proposons, dans notre contribution, d'explorer ce lien paradoxal entre un lieu - la ville -, la fiction - et plus précisément la littérature urbaine $\mathrm{du} \mathrm{XIX}^{\mathrm{e}}$ siècle - et la vérité d'une fiction paradoxe poétique s'il en est -, pour mettre en évidence ce qui s'inscrit en filigrane dans cette relation tri-partite, à savoir le mensonge, l'autre face de la vérité et de la fiction. Nous nous intéresserons d'abord à la longue tradition de la littérature urbaine qui illustre la citation barthésienne et où le lieu de la fiction - la ville - détient ou déclenche effectivement une vérité, politique, sociologique ou sentimentale qu'il faut trouver derrière l'apparence souvent mensongère que représente le paysage urbain. Car historiquement, dès le $\mathrm{XVIII}^{\mathrm{e}}$ siècle, les grandes entreprises poétiques, qu'elles relèvent de la chronique urbaine feuilletonesque ou du roman, s'attachent à découvrir derrière le visage trompeur de la ville, et notamment de Paris, une vérité cachée et une promesse de connaissance que les habitants des grandes villes ont de plus en plus de mal à saisir en raison de la complexité croissante de la topographie urbaine ${ }^{2}$. Ainsi, chez Balzac, les protagonistes doivent déchiffrer la ville dans sa complexité sociale et érotique afin de connaître sa vérité et de pouvoir ainsi prétendre au pouvoir mondain et sexuel. La physionomie de la ville dévoile ses mystères aux yeux de celui qui sait la lire correctement et pour qui cette vérité urbaine est à son tour synonyme de conquête personnelle, d'ascension sociale et de gratification sexuelle. Si pour l'auteur de La Comédie humaine, Paris est donc avant tout une formidable énigme en attente d'une solution qui permette de distinguer le physionomiste perspicace (l'écrivain) d'un observateur naïf (le lecteur), il ne fait pourtant pas de doute que la vérité du lieu peut et doit se dévoiler.

Dans l'œuvre de Flaubert, c'est l'inverse qui se produit, comme nous allons

1 Roland Barthes, Le Degré zéro de l'écriture, Paris, Éditions du Seuil, 1972, p. 158.

2 Cf. Priscilla Parkhurst ferguson, Paris as a Revolution. Writing the Nineteenth-Century City, Berkeley/ Los Angeles/ London, University of California Press, 1994; Christopher Prendergast, Paris and the Nineteenth Century, Oxford/ Cambridge Mass., Blackwell, 1995; Pierre Citrons, La Poésie de Paris dans la littérature française de Rousseau à Baudelaire, 2 vol., Paris, Éditions de Minuit, 1961; Karl-Heinz Stierle, La Capitale des signes. Paris et son discours, Paris, Éditions de la Maison des Sciences de l'Homme, 2001. 
le démontrer dans notre deuxième partie: le lieu d'une fiction y est toujours un mensonge que la poétique flaubertienne parvient à conjurer d'une façon magistrale, rompant ainsi avec les codes de la littérature urbaine, tels que nous les connaissons depuis la Révolution française. On assiste, chez Flaubert, et notamment dans $L^{\prime}{ }^{\prime}$ ducation sentimentale que nous étudierons dans la troisième partie, à une mise en question radicale de l'espace urbain comme lieu de véracité. La ville devient alors synonyme de mensonge, de tromperie, d'inauthenticité. Elle engendre un texte qui déforme la vérité et illustre le décalage entre émotion et expression - constitutif de la notion de mensonge telle que nous la comprenons - et cela au niveau scénarique, narratologique et sémiotique. Pour terminer, nous allons nous interroger sur les raisons politiques et épistémologiques qui peuvent expliquer les changements intervenus dans la poétique de la ville et le regard de l'écrivain urbain.

\section{I}

Historiquement, le déchiffrement du paysage urbain en vue d'une vérité à découvrir se trouve au cœur du projet littéraire lié à la ville. Cette ambition était revendiquée par les écrivains-flâneurs comme Louis Sébastien Mercier et fonde le genre des chroniques urbaines ${ }^{3}$. L'essor de cette littérature physionomiste que sont les tableaux de Paris est lié à la croissance et aux bouleversements politiques et urbains, qui, à partir du XVIII ${ }^{\mathrm{e}}$ siècle, ont tendance à rendre la ville étrangère et incompréhensible aux yeux de ses propres habitants, un fait que Mercier ne cesse de souligner. Devenant de plus en plus complexe, la ville nécessite désormais un travail d'interprétation et de déchiffrement que les écrivains urbains ont vocation à fournir afin d'expliquer à leurs lecteurs la vérité de la ville - sa configuration sociale, sa fonction politique symbolique, sa signification amoureuse et érotique. Si les auteurs feuilletonistes se sont arrogé le rôle du guide virgilien qui présente un tableau urbain intelligible, les grands romanciers de Paris comme Balzac et Hugo, pour leur part, s'approprient une fonction encore plus impérieuse et triomphante: celle de l'artiste tout-puissant qui n'interprète pas seulement la ville réelle, mais la recrée en lui superposant un double imaginaire d'autant plus véridique ${ }^{4}$.

L'analyse de Ferragus, roman parisien d'amour et d'espionnage que Balzac publie en 1833 met en évidence le jeu de miroirs entre vérité et mensonge constitutif de la littérature urbaine ${ }^{5}$.

3 Cf. Eckhardt KöHn, Straßenrausch. Flanerie und kleine Form. Versuch zur Literaturgeschichte des Flaneurs bis 1933, Berlin, Das Arsenal, 1989; Lewis Mumford, La Cité à travers l'histoire, Paris, Éditions du Seuil, 1964; Joachim Schlör, Nachts in der großen Stadt. Paris, Berlin, London 1840-1930, München, Deutscher Taschenbuch Verlag, 1991; Philippe Hamon, Imageries. Littérature et image au XIX $X^{e}$ siècle, Paris, Corti, 2001.

4 Pour une analyse de l'instabilité des signes et de la double métamorphose de l'écrivain par la ville et de la ville par le regard de l'écrivain, voir Pierre Sansot, Poétique de la ville, Paris, Payot, 2004.

5 Il convient de rappeler ici l'appartenance de Ferragus à L'Histoire des Treize. Le lien qu'entretient ce roman avec La Duchesse de Langeais et La Fille aux yeux d'or explique en partie l'atmosphère mystérieuse dans laquelle baigne le texte. La dimension fantastique est, certes, quelque peu désamorcée par la préface de l'Histoire des Treize: «Ferragus est un premier épisode qui tient par d'invisibles liens 
L'auteur de La Comédie humaine affirme la nature insaisissable et opaque de la capitale qui en fait un lieu de mensonge pour le commun des mortels. Or, cela permet surtout de mettre en valeur les connaissances du romancier et son regard supérieur, capable de cerner la vérité derrière les apparences mensongères. Car, comme le souligne Balzac dans sa modestie habituelle, l'écrivain appartient à cette espèce rare des flâneurs-artistes capables de "surprendre le sens caché dans cet immense assemblage de figures, de passions et d'événements ${ }^{6}{ }$ " que constitue la vie, et à plus forte raison la vie parisienne. Cette connaissance intime de la ville permet de distinguer la vérité de l'illusion et du mensonge et d'avoir une vue d'ensemble qui échappe aux non-initiés. En revanche, pour les initiés, [les écrivains] « Paris est une créature; chaque homme, chaque fraction de maison est un lobe du tissu cellulaire de cette grande courtisane de laquelle ils connaissent parfaitement la tête, le cœur et les mœurs fantasques. Aussi, ceux-là sont-ils les amants de Paris7. » Passons, pour l'instant, sur l'érotisation de la ville et du regard de celui qui essaie de lui arracher ses vérités secrètes et tenons-nous en à constater qu'être le prétendant de la courtisane Paris implique de maîtriser cette " science des distinctions et des identifications ${ }^{8}$ " à laquelle Balzac excelle dans la Comédie humaine. Une telle science permet de cerner les nuances infimes et à peine perceptibles qui ont désormais remplacé les signes sociaux distinctifs, de façon à découvrir des vérités cachées ou des mensonges dissimulés. Cela dit, tout le roman s'attache à montrer combien il est facile de se tromper dans l'interprétation sociale et le déchiffrement urbanistique (les deux étant intimement liés). Les conséquences peuvent alors s'avérer tragiques. Ferragus nous fait ainsi assister à une scène de déduction trompeuse que l'on peut considérer comme paradigme même de l'influence mensongère qu'exercent la physionomie urbaine et le désir de l'homme sur la perception: lors d'une course dans Paris, Auguste de Maulincour, jeune homme bien comme il faut, croise par hasard les pas de Claire Desmarets, la femme mariée et vertueuse dont il est secrètement amoureux, et cela dans la rue Soly, une des rues les plus mal famées de Paris: « Il connaissait Paris; et sa perspicacité ne lui permettait pas d'ignorer tout ce qu'il y avait d'infamie possible pour une femme élégante, riche, jeune et jolie, à se promener là, d'un pied criminellement furtif. Elle, dans cette crotte, à cette heure ${ }^{9} ! »$. Ce paragraphe, tout comme les pages qui le précèdent et le suivent, repose sur une mise en scène subtile. Car le narrateur ne cesse de cautionner et la réaction du jeune homme (le choc, suite à la rencontre d'une femme de bonne réputation dans un endroit déshonorant), et sa raison (l'effet contagieux de la rue). Non seulement, le point de vue du paragraphe en

\footnotetext{
à l'Histoire des Treize, dont la puissance naturellement acquise peut seule expliquer certains ressorts en apparence surnaturels » (Honoré de BaLzac, Histoire des Treize, éd. par Pierre-Georges CasteX, Paris, Garnier, 1966, p. 12). Mais cette explication narrative de l'ambiance énigmatique qui règne dans le roman ne parvient aucunement à neutraliser la sensation étrange et inquiétante qui surgit à sa lecture.

6 Honoré de Balzac, La Comédie humaine, t. I, éd. par Pierre-Georges Castex, Paris, Gallimard (coll. «Bibliothèque de la Pléiade »), 1976, p. 11.

7 Honoré de Balzac, La Comédie humaine, t. V, éd. par Pierre-Georges CAstex, Paris, Gallimard (coll. « Bibliothèque de la Pléiade »), 1977, p. 795.

8 Jacques NeEFs, «L'épreuve littéraire de la ville», Temps Libre. Crise de l'urbain. Futur de la ville, $\mathrm{n}^{\circ} 11,1985$, p. 100.

9 Balzac, La Comédie humaine, t. V, p. 796-97.
} 
question est clairement attribuable au narrateur jusqu'au moment où l'exclamation introduit la voix du protagoniste, mais de plus, avant même de nous montrer la scène de rencontre néfaste, le narrateur prépare le terrain: "Il est des rues, ou des fins de rue, il est certaines maisons, inconnues pour la plupart aux personnes du grand monde, dans lesquelles une femme appartenant à ce monde ne saurait aller sans faire penser d'elle les choses les plus cruellement blessantes ${ }^{10}$. $\gg$ Au cas où nous, lecteurs innocents et pudiques, n'aurions pas encore compris de quoi il était question, le narrateur précise :

Si cette femme est riche, si elle a voiture, si elle se trouve à pied ou déguisée, en quelques-uns de ces défilés du pays parisien, elle y compromet sa réputation d'honnête femme. Mais si, par hasard, elle y est venue à neuf heures du soir, les conjectures qu'un observateur peut se permettre deviennent épouvantables par leurs conséquences ${ }^{11}$.

Comment alors ne pas comprendre la réaction du jeune héros, après une telle introduction de la part du narrateur? Comment ne pas partager son indignation et, avec un peu d'empathie, même son orgueil blessé d'amoureux qui a renoncé, là où d'autres, apparemment, avaient moins de scrupules? Le lecteur est d'autant plus prêt à adopter le regard du jeune homme, que tout suggère qu'il dispose bel et bien de ce regard délicat et perspicace qui permet de saisir l'espace urbain et social et les femmes qui le fréquentent. Le narrateur nous en donne même un exemple: « La lueur vacillante que projetait le vitrage d'une boutique de cordonnier illumina soudain, précisément la chute des reins, la taille de la femme qui se trouvait devant le jeune homme. Ah! certes, elle seule était ainsi cambrée! Elle seule avait le secret de cette chaste démarche qui met innocemment en relief les beautés des formes les plus attrayantes ${ }^{12} \gg$. Malgré la tournure fantasmatique que prend l'observation de Maulincour, il a effectivement, aux yeux du lecteur, livré une preuve de son œil sagace, capable de reconnaître la silhouette de la femme qu'il désire. En laissant le lecteur partager l'interprétation de Maulincour, soigneusement préparée et rendue encore plus convaincante par la réflexion du narrateur sur la physionomie des rues, Balzac fait de nous un complice du drame qui se déroulera. Car loin de retrouver quelque vil amant dans un lieu mal famé, Mme Desmarets ne fait que rendre une visite inspirée par l'amour filial à son père, Ferragus, ancien forçat échappé du bagne. Balzac prouve au lecteur que lui aussi se serait trompé, que lui aussi aurait été dupe d'une lecture hâtive et erronée et surtout, que lui aussi a besoin du narrateur comme guide et interprète. Car c'est bien de cela qu'il s'agit: démontrer au lecteur que l'écrivain seul détient les clés du mystère que forment Paris et le récit qui y prend place, que lui seul sait reconnaître la vérité, là où le lecteur ne voit que mensonge. Par ce geste narratif suprême, Balzac se situe pleinement dans la tradition des tableaux urbains et reprend à son compte la posture que L. S. Mercier y avait

${ }^{10}$ Ibid., p. 795. Cf. Gérard Genette et ce qu'il appelle « le démon explicatif» chez Balzac («Vraisemblance et motivation », in Gérard Genette, Figures II, Paris, Seuil (coll. « Points »), 1979, p. 79). Cette posture narrative qui imprime sa marque à Ferragus n'est pas limitée au traitement de Paris dans les fictions balzaciennes (nous remercions Hélène Barrière pour ses précieuses remarques sur la poétique balzacienne).

11 Ibid., p. 795-96.

12 Ibid., p. 798. 
adoptée. Le début du roman ne décline pas seulement la devise du flâneur, ce «j’y suis allé pour vous ${ }^{13} »$ que fait valoir Mercier quand il s'aventure dans les bas-fonds de Paris, mais s'attache surtout à montrer au lecteur que même s'il avait osé mettre les pieds dans une rue pareille, il aurait été incapable d'interpréter correctement la scène. En mettant en scène un " vaste appareil d'interprétation des singularités ${ }^{14}$ », Balzac signifie au lecteur que la tâche qui incombe au flâneur ne se résume plus à côtoyer les lieux de mystères et de dangers à la place du lecteur, mais consiste bel et bien à les déchiffrer et à les expliquer aux non-initiés. Dès lors, la réflexion sur la nature trompeuse de Paris prend tout son sens.

\begin{abstract}
Il se rencontre dans Paris des effets de nuits singuliers, bizarres, inconcevables. Ceuxlà seulement qui se sont amusés à les observer savent combien la femme y devient fantastique à la brune [...]; enfin les clartés incertaines d'une boutique ou d'un réverbère donnent à l'inconnue un éclat fugitif, presque toujours trompeur, qui réveille, allume l'imagination et la lance au-delà du vrai. Les sens s'émeuvent alors, tout se colore et s'anime; la femme prend un aspect tout nouveau; son corps s'embellit; par moments ce n'est plus une femme, c'est un démon, un feu follet qui vous entraîne par un ardent magnétisme ${ }^{15}$.
\end{abstract}

Situé juste avant la rencontre rue Soly, ce passage fait figure d'avertissement quant au danger d'une perception erronée sous l'influence fallacieuse de la ville et, bien sûr, de la femme et de son désir pour elle. L'évocation du Paris nocturne est empreinte d'une atmosphère mystérieuse où toute vision est sujette à confusion, où toute apparition est trompeuse et potentiellement mensongère. Dans la plus pure tradition de la littérature fantastique, la femme apparaît, suite à la nature mystérieuse de la ville, sous les traits d'un démon qui charme et entraîne l'aventurier urbain. L'énigme de Paris et celle de la femme se confondent et le narrateur s'apprête à les résoudre pour nous. La clairvoyance affichée du narrateur qui perce les secrets de la ville et du sexe, assimilant par cela la physionomie urbaine à un corps de femme à explorer, caractérise la représentation de Paris dans Ferragus, mais aussi dans toute l'œuvre balzacienne. La Comédie humaine procède à une appropriation de l'espace urbain par le récit, et s'avère une formidable entreprise de contrôle narratif et symbolique et de rétablissement de la vérité ${ }^{16}$. En cela, elle participe à un discours

${ }^{13}$ Louis Sébastien Mercier, « Cabaret borgnes », in Le Tableau de Paris, t. I, éd par Jean-Claude Bonnet, Paris, Mercure de France, 1994, p. 179.

14 NeEFs, « L'épreuve littéraire de la ville », p. 100.

15 Balzac, La Comédie Humaine, t. V, p. 797.

${ }^{16} \mathrm{La}$ lecture balzacienne de la physionomie urbaine et le regard perspicace du narrateur qui sait reconnaître la physiologie des passants se situent dans la continuité du genre des physiologies, en vogue pendant les années quarante du XIX ${ }^{\mathrm{e}}$ siècle. Walter Benjamin a souligné le rôle sécurisant et pédagogique dévolu à ces textes qui réagissent à la nature inquiétante de la grande ville (Walter BENJAMIN, « Le Paris du Second Empire chez Baudelaire », in Charles Baudelaire. Un poète lyrique à l'apogée du capitalisme, Paris, Payot, 1979, p. 57-63.) Celle-ci devenant de plus en plus menaçante, les lecteurs ont besoin d'être rassurés par une représentation simple et reconnaissable des types urbains. Or, l'émergence du roman policier montre que le potentiel menaçant de la ville ne pouvait plus être contenu dans des esquisses de caractères facilement identifiables. La diversité urbaine demande désormais une véritable enquête policière, menée par un flâneur qui se fait détective. Physiologies et roman policier, malgré leur approche diamétralement opposée, témoignent de la même nécessité de prendre acte de la complexification de l'espace urbain. La Comédie humaine se nourrit des deux traditions et, de façon presque perverse, pousse 
littéraire qui affirme sa maîtrise sur le paysage de la ville par un regard qui accède à la connaissance et perce le mensonge.

\section{II}

Dans notre deuxième partie sur l'écriture de la ville chez Flaubert, nous allons étudier comment l'auteur de L'Éducation sentimentale pratique une poétique urbaine qui, tout en reprenant un certain nombre de motifs et de figures narratives propres à ce genre, met en relief l'illisibilité croissante de la texture urbaine, de façon à faire de la ville un lieu de tromperie et de mensonge par excellence. Qu'est-ce qui nourrit la nature foncièrement mensongère de la ville? Quels procédés narratifs ou symboliques font que Paris devient le lieu suprême du mensonge dans l'œuvre flaubertienne? Voilà les questions que nous allons aborder en suivant Frédéric Moreau dans ses pérégrinations dans la ville lumière.

À la lecture de L'Éducation sentimentale, on constate aisément que la vision unifiée qui caractérisait la représentation de Paris dans les écrits de Balzac ou de Hugo et qui, in fine, aboutissait à une vérité sociale, politique ou imaginaire se fissure. Le narrateur flaubertien dédaigne le regard surplombant et auctorial qui pourrait produire une vision d'ensemble significative. Flaubert se refuse à déchiffrer la texture urbaine à la place de son protagoniste ou à donner des indications qui permettraient aux lecteurs et aux personnages de sortir du labyrinthe de la ville, de faire la distinction entre vérité ou mensonge. La capitale paraît alors plus difficilement cernable que dans les enquêtes urbaines mises en scène par Balzac. Car, grâce aux connaissances supérieures du narrateur balzacien, les énigmes citadines et urbaines de la Comédie humaine finissent toujours par être résolues, de façon à souligner la maîtrise narrative de l'écrivain. Par rapport au fonctionnement d'auteurs comme Balzac, l'autorité symbolique que Flaubert exerce sur le texte urbain et le texte romanesque se déplace. Ce n'est plus dans l'acte de déchiffrement que la maîtrise de l'écrivain s'affirme, mais dans la représentation accomplie de l'illisibilité urbaine et de la ville comme mensonge. Le procédé le plus important qui contribue à faire de la physionomie urbaine une topographie trompeuse et mensongère, réside dans la narrativisation spatiale des désirs et des sentiments des protagonistes. La ville ne signifie plus une vérité, mais change en fonction des états d'âme du protagoniste. Ainsi, dans L'Éducation sentimentale, Paris matérialise les espoirs de Frédéric Moreau, elle donne corps à son ennui et elle scénarise ses trahisons subies et commises. Cette contamination de l'espace par les émotions du personnage principal, sa représentation par le filtre de ses perceptions font de la ville une topographie du désir. La ville est mensonge car elle est un lieu de désir. $\mathrm{Ou}$, autrement dit, l'inscription du désir dans la ville fait de l'espace urbain un texte foncièrement mensonger, car, bien entendu, pour Flaubert, le désir relève au mieux de l'illusion, mais la plupart du temps tout simplement du mensonge. Dans Madame Bovary, cette inscription d'un désir fallacieux dans une topographie qui ne

à l'extrême l'aspect opaque de la ville que seul son narrateur sait éclaircir. 
l'est pas moins se cristallise d'abord dans l'achat du plan de la ville, permettant à Emma de s'évader de sa morne vie provinciale vers Paris, incarnation ultime de cette 《 attirante fantasmagorie des réalités sentimentales ${ }^{17}$ » qu'elle désire si ardemment et qui finira par lui faire perdre réputation, économies et même la vie.

Elle s'acheta un plan de Paris, et, du bout de son doigt, sur la carte, elle faisait des courses
dans la capitale. Elle remontait les boulevards, s'arrêtant à chaque angle, entre les lignes
des rues, devant les carrés blancs qui figurent les maisons. Les yeux fatigués à la fin, elle
fermait ses paupières, et elle voyait dans les ténèbres se tordre au vent des becs à gaz,
avec des marche-pieds de calèches, qui se déployaient à grand fracas devant le péristyle
des théâtres ${ }^{18}$.

Illustrant la puissance de projection et la qualité fantasmagorique des plans de ville dont « la force invisible n'est pas [...] moins importante que la vie propre à l'agencement de la ville ${ }^{19}{ }^{\prime}$ comme le souligne Walter Benjamin dans Le Livre des Passages, ce paragraphe met en évidence le pouvoir hypnotique et érotique qui émane du nom de la ville et de ses rues et qu'Emma subit à maintes reprises: «Comment était ce Paris? Quel nom démesuré! Elle se le répétait à demi-voix, pour se faire plaisir; il sonnait à ses oreilles comme un bourdon de cathédrale, il flamboyait à ses yeux jusque sur l'étiquette de ses pots de pommade ${ }^{20}$. » La sensualité du nom de la ville conjure des images de rencontres galantes et des jouissances urbaines que les prés normands et le mari rustre d'Emma ne peuvent lui promettre. Il n'est donc pas étonnant que la seule évocation du nom de la ville suffise à faire perdre la tête et le sens du devoir conjugal à Emma. Car nous trouvons, dans Madame Bovary, la scène matricielle qui met en scène la fonction et la symbolique mensongère de la ville dans l'œuvre flaubertien. Il s'agit de la rencontre fatidique entre Emma et Léon à Rouen, qui à la simple évocation du nom de la capitale amène l'héroïne du roman au passage à l'acte adultère et Flaubert au tribunal pour « outrage à la morale publique et religieuse et aux bonnes mœurs ${ }^{21} »$. Emma et Léon, après s'être tourné autour depuis un certain temps et avoir épuisé les plaisirs platoniques des échanges spirituels sur la poésie, le chant lyrique, les sentiments héroïques et les amours chevaleresques,

17 Gustave Flaubert, Madame Bovary, éd. par Claudine Gothot-Mersch, Paris, Bordas, 1990, p. 42.

18 Ibid., p. 59.

19 Walter Benjamin, Paris, Capitale du XIX $X^{e}$ siècle, Le Livre des Passages, Paris, Les Éditions du Cerf, 1993, p. 533. Une analyse du rôle fantasmagorique de la ville chez Benjamin, intimement lié à la figure du flâneur qui se fait chiffonnier de l'histoire, dépasserait le cadre ce cet article. Cf., à ce propos, Walter Benjamin et Paris, éd. par Heinz Wismann, Paris, Les Éditions du Cerf, 1983; Christine SCHMIDER (en collaboration avec Michael WeRNER), « Das Baudelaire-Buch », in Benjamin Handbuch. Leben - Werk - Wirkung, éd. par Burkhardt Lindner, Stuttgart, Metzler, 2006, p. 569-584 ; Susan BucKMORSs, The Dialectics of Seeing. Walter Benjamin and the Arcades Project, Cambridge Mass./ Londres, The MIT Press, 1995 ; Michael OPITZ, « Lesen und Flanieren. Über das Lesen von Städten, vom Flanieren der Bücher », in Aber ein Sturm weht vom Paradies her. Texte zu Walter Benjamin, Leipzig, Reclam, 1992; Rainer Rochlitz, "La ville de Paris, forme symbolique », Revue d'esthétique, hors série Walter Benjamin, Paris, Éditions Jean-Michel Place, 1990; Heiner Weidmann, Flanerie, Sammlung, Spiel. Die Erinnerung des 19. Jahrhunderts bei Walter Benjamin, München, Fink, 1992.

${ }^{20}$ Flaubert, Madame Bovary, p. 59.

${ }^{21}$ Gustave Flaubert, Euvres, t. I, éd. par René Dumesnil et Albert Thibaudet, Paris, Gallimard (coll. « Bibliothèque de la Pléiade »), 1951. Voir l’appendice : « Réquisitoire ». 
se trouvent à Rouen devant la cathédrale, pour un rendez-vous qui doit, pour Emma ayant apporté une lettre de rupture, mettre un terme à leur relation et dont Léon au contraire espère qu'il lui permettra enfin de conclure. À la fin d'une interminable visite de la cathédrale conduite par un guide zélé, Léon, n'en pouvant plus de voir différer le plaisir promis, parvient enfin à héler un fiacre. Pourtant, Emma, tout à son rôle de chaste épouse, résiste: «Ah! Léon !... Vraiment... je ne sais... si je dois... ! Elle minaudait. Puis, d'un air sérieux: C'est très inconvenant, savez-vous ${ }^{22}$ ? ». Finalement, ce n'est ni le charme du jeune homme, ni l'amour qu'elle lui porte ou la promesse d'une gratification sexuelle incomparable à celle que le pauvre bougre Charles peut lui garantir qui lui font franchir le pas. C'est la réponse de Léon, qui avance l'argument imparable: «En quoi? répliqua le clerc. Cela se fait à Paris ${ }^{23}$ ! ». Il est évident que l'on ne peut rien opposer à pareil raisonnement. Emma succombe à la tentation et monte dans le fiacre qui arrive opportunément.

\section{III}

Paris comme lieu ultime de la trahison, du mensonge et de la tromperie, c'est aussi ce qui nous est donné à voir dans L'Éducation sentimentale, à travers les yeux d'un protagoniste qui y projette ses rêves, ses fantasmes et ses désirs. La ville y devient ainsi un espace hautement fantasmagorique. Faisant miroiter des promesses sentimentales et politiques à Frédéric et à sa génération, la capitale déçoit pourtant les espoirs qu'elle engendre, et s'avère un lieu d'illusion. Peuplé de figures trompeuses qui cristallisent les rêves collectifs et alimentent une économie du désir, le Paris de Flaubert se présente comme un monde marchand, illusoire et ambigu, propre à nourrir les mensonges du jeune héros et à le tromper à son tour.

Frédéric Moreau, en proie au même sentimentalisme éculé qu'Emma, n'a pas beaucoup de convictions. Or, il y a bien une chose dont il est certain, tout comme Emma, c'est qu' «il n'existait au monde qu'un seul endroit pour [...] faire valoir [les secrètes opulences de sa nature]: Paris! car, dans ses idées, l'art, la science et l'amour [...] dépendaient exclusivement de la capitale ${ }^{24} \gg$. Seulement, tout au long du roman, cette promesse qu'incarne la ville ne cesse de se dérober. L'art, la science et l'amour fuient le héros qui s'avère totalement incapable de déchiffrer la physionomie urbaine, de sonder sa stratification sociale, de saisir le moindre indice qui lui permettrait de maîtriser la ville et de satisfaire ses ambitions et ses désirs. C'est comme si la ville, personnage maléfique et trompeur, se moquait de lui, s'efforçait de l'induire en erreur, le faisait tourner en rond pour finir par le perdre. «Dramatisé comme espace labyrinthique de recherches, de conflits, de pertes, de rencontres ${ }^{25} »$, l'univers de la capitale possède un caractère dédaléen qui structure

${ }^{22}$ Flaubert, Madame Bovary, p. 249.

23 Ibid.

${ }^{24}$ Gustave Flaubert, L'Éducation sentimentale, éd. par Claudine Gothot-Mersch, Paris, Flammarion, 1985, p. 144-145.

25 Jacques NeEFs, «Description de l'espace et espaces de socialité », Histoire et langage dans L'Éducation sentimentale de Flaubert, Paris, Société des Études romantiques, C.D.U.-SEDES, 1981, p. 116. 
l'intrigue romanesque. La figure du labyrinthe qui, chez Balzac encore, fonctionnait comme vecteur d'une initiation reposant sur une quête et permettant au jeune héros comme Rastignac d'accomplir son utopie personnelle, se transforme, dans $L$ 'Éducation sentimentale, en figure de l'immanence et de la répétition. En rythmant le roman par une multitude de scènes qui se font écho, comme les courses fiévreuses de Frédéric dans la ville nocturne ou ses balades au bras de différentes femmes, Flaubert agence l'espace citadin comme une suite de répétitions et de variantes, de façon à véhiculer l'intrigue qui, en fait, n'avance pas. Les confrontations imprévues entre les femmes-rivales ${ }^{26}$, les innombrables rencontres fortuites dans les rues, les apparitions gênantes de l'ami de Frédéric, Deslauriers, qui rappelle les promesses d'argent non tenues et les nombreux rendez-vous manqués, scénarisent le texte urbain et déçoivent les espoirs du personnage qui se retrouve toujours au même endroit, malgré les efforts désespérés pour atteindre son but. Paris est narrativisé comme un lieu de la tromperie où la promesse d'un événement n'est qu'illusion, où les rues ne sont que des impasses et le désir de la femme aimée devient mensonge. La ville égare et trompe, elle « contrecarre [...] les ambitions des personnages et tisse d'imperceptibles ironies ${ }^{27} »$, comme quand elle fait échouer la déclaration d'amour de Frédéric: «L'occasion était bonne, le temps pressait. Il se donna jusqu'à la rue de Richelieu pour déclarer son amour. Mais, presque aussitôt, devant un magasin de porcelaine, elle s'arrêta net, en lui disant: Nous y sommes, je vous remercie! À jeudi, n'est-ce pas, comme d'habitude ${ }^{28}$.»

La ville devient aussi, dans L'Éducation sentimentale un lieu de substitution, voire de prostitution ${ }^{29}$. Le contraste entre les endroits toujours identiques et l'échange continuel des femmes accentue l'ambiance de promiscuité et de mensonge qui règne dans Paris :

Quelques fois, il se réveillait le cœur plein d'espérance, s'habillait soigneusement comme pour un rendez-vous, et il faisait dans Paris des courses interminables. À chaque femme qui marchait devant lui, ou qui s'avançait à sa rencontre, il se disait: La voilà: C'était chaque fois une déception nouvelle. L'idée de Mme Arnoux fortifiait ses convoitises. Il la trouverait peut-être sur son chemin ${ }^{30}$.

Or, les chemins dans Paris ne font venir, vers lui, que d'autres femmes lui faisant miroiter l'image de Marie Arnoux et suscitant un désir polymorphe et mobile: « Les prostituées qu'il rencontrait aux feux du gaz, les cantatrices poussant leurs roulades, les écuyères sur leurs chevaux au galop, les bourgeoises à pied, les grisettes à leur fenêtre, toutes les femmes lui rappelaient celle-là, par des similitudes ou par des

${ }^{26}$ Par exemple à l'hippodrome lorsque Frédéric, fort gêné car accompagné par Rosanette, rencontre Mme Arnoux et les Dambreuse (FLAUBERT, L'Éducation sentimentale, p. 269-270.), ou chez Mme Arnoux quand Rosanette interrompt les déclarations mutuelles (ibid., p. 434), ou encore à la saisie du mobilier des Arnoux qui donne lieu à une confrontation entre Marie Arnoux et Mme Dambreuse (ibid., p. 495).

${ }^{27}$ Michael Wetherill, "Paris dans L'Éducation sentimentale », in Marie-Claire BANCQUART, Flaubert, la femme, la ville, Paris, Presses Universitaires de France, 1980, p. 132.

28 Flaubert, L'Éducation sentimentale, p. 119.

${ }^{29}$ Cf. Marie-Claire BANCQUART, «L'espace urbain de L'Éducation sentimentale: intérieursextérieurs », in BANCQUART, Flaubert, la femme, la ville, p. 148-151.

${ }^{30}$ Flaubert, L'Éducation sentimentale, p. 73. 
contrastes violents $^{31}$. » La nature unique de celle qui faisait « le point lumineux ${ }^{32}$ » de son existence se perd au profit d'une multiplication illimitée et vénale, contaminant l'idéal amoureux qui sous l'influence pernicieuse de la ville se dégrade pour devenir mensonge. Ainsi, au retour de son exil forcé à Nogent, Frédéric retrouve Paris plein d'espoir et d'un esprit conquérant. Ayant hérité la fortune de son oncle qui jusque-là refusait de le soutenir, il retourne à Paris pour enfin y faire carrière, briller en société et séduire la femme de son rival Arnoux : « Frédéric alla de l'estaminet chez Arnoux comme soulevé par un vent tiède et avec l'aisance extraordinaire que l'on éprouve dans les songes ${ }^{33}$. \ Cependant, l'élan que Frédéric ressent à l'idée de retrouver la femme aimée n'est pas fait pour durer. Ayant enfin retrouvé sa bien aimée qui entretemps a déménagé, il subit une déception cruelle:

Frédéric s'était attendu à des spasmes de joie; - mais les passions s'étiolent quand on les dépayse, et, ne retrouvant plus Mme Arnoux dans le milieu où il l'avait connue, elle lui semblait avoir perdu quelque chose, porter confusément comme une dégradation, enfin n'être pas la même. Le calme de son cœur le stupéfiait ${ }^{34}$.

Une fois de plus, la satisfaction du désir échoue sous l'impulsion de la ville qui dérobe l'objet désiré, le déplace en son sein, de façon à modifier le scénario fantasmé de Frédéric.

À l'évidence, notre héros ne parvient pas à atteindre ses buts, que ce soit dans le domaine de l'amour, de la carrière artistique ou autre, ou dans la conquête du pouvoir. À mille lieues d'un Rastignac, Frédéric peine à agir, à saisir les occasions et à lire les signes qui lui permettraient de distinguer le vrai du faux, de repérer le mensonge et de se placer comme il le faut au cœur d'une physionomie urbaine indéchiffrable. Flaubert multiplie d'ailleurs les allusions explicites ou implicites aux codes balzaciens du roman et aux scènes archétypales de la vie parisienne que l'on y trouve. Ainsi, la scène de l'enterrement du banquier Dambreuse sur les hauteurs du cimetière Lachaise ou les innombrables scènes qui nous montrent Frédéric en train de contempler la ville du haut de son balcon, repérant l'endroit ou habite la femme aimée, parodient le fameux "à nous deux, Paris» de Rastignac dans le Père Goriot. Ces tableaux panoramiques construisent une vision de la capitale où la conquête de la ville et de la femme se superposent, mais qui joue avec l'intertexte balzacien pour mieux le déconstruire ${ }^{35}$. La maitrise de la topographie du désir que représente Paris, ce geste balzacien par excellence, est irréalisable pour Frédéric et cela pour deux raisons: Frédéric n'a ni le courage nécessaire pour s'approprier la ville, ni la perspicacité qui permet aux héros balzaciens de lire les signes

31 Ibid., p. 119.

32 Ibid., p. 55.

33 Ibid., p. 162.

${ }^{34}$ Ibid., p. 163.

${ }^{35}$ En ce qui concerne les rapports d'intertextualités et d'influences entre Balzac et Flaubert, voir aussi: Graham FALCONER, "Le travail de débalzaciénisation dans la rédaction de Madame Bovary », in Gustave Flaubert 3. Lettres Modernes, Paris, Minard, 1988, p. 123-156; Guy SAGnes, « De Balzac à Flaubert: l'enfance de Deslauriers d'après le manuscrit de L'Éducation sentimentale », Littératures, $\mathrm{n}^{\circ} 2$, automne 1989, p. 17-27; Alain VAILlIANT, « Conversation sous influence - Balzac, Baudelaire, Flaubert, Mallarmé », Romantisme, Influence, $\mathrm{n}^{\circ}$ 98, 1997, p. 97-110. 
distinctifs de la vie sociale dans la capitale et de s'imposer au milieu des mensonges. Le meilleur exemple en est le projet de séduction avorté qui le fait tourner autour de Mme Dambreuse, la richissime femme du banquier. Le scénario, tout en imposture et mensonge, est on ne peut plus balzacien: lassé par les tentatives vaines de séduire Mme Arnoux dont la chasteté niaise lui résiste depuis des années et ennuyé par le ménage avec l'appétissante mais vulgaire cocotte Rosanette, Frédéric se donne un défi tout balzacien - la conquête de Mme Dambreuse dont le mari possède « deux coffres monstrueux, dressés dans les encoignures. [Frédéric] se demandait combien de millions pouvaient y tenir ${ }^{36} »$. Il va de soi que la promesse de cette richesse immense donne un attrait particulier à la femme du banquier et fait mieux passer son physique sec et sa nature revêche, au moins jusqu'au dénouement tragique. Car dès le début, Frédéric se leurre sur toute la ligne et s'empêtre dans le tissu de tromperies et de manipulations que forme le triangle amoureux. Ainsi, son amour pour la femme du banquier se nourrit de la richesse qu'elle lui fait miroiter et du dépit qu'il éprouve par rapport à la poursuite infructueuse de Mme Arnoux. Mme Dambreuse quant à elle veut prendre du bon temps, mais surtout punir son mari de lui avoir imposé, dans leur foyer, la présence de sa fille illégitime. Et le banquier lui-même ne fait semblant d'accepter les escapades de sa femme que pour mieux mûrir sa vengeance et la déshériter à sa mort. Frédéric, dupe de tous les mensonges, se retrouve finalement devant des coffres vides: ceux du banquier ne contiennent pas le testament qui aurait fait de lui un homme enfin riche, et la femme qui aurait dû le combler dans ses ambitions sociales et le faire accéder à la haute société se montre, une fois désargentée, sous son vrai jour - vaine et superficielle. Dans l'une des rares interventions directes du narrateur, celui-ci commente alors : « les cœurs des femmes sont comme ces petits meubles à secret, pleins de tiroirs emboîtés les uns dans les autres; on se donne du mal, on se casse les ongles, et on trouve au fond quelques fleurs desséchées, des brins de poussières - ou le vide ${ }^{37}$ ! » Loin du héros balzacien qui manipule habilement les mensonges pour s'en servir dans sa quête du pouvoir, le protagoniste flaubertien n'arrive jamais à les percer et en finit toujours victime, sans que nous, lecteurs, ne compatissions. Car Frédéric n'est pas seulement dupe de tous les mensonges, il est surtout terriblement faible et lâche, à l'opposé du jeune héros conquérant auquel La Comédie humaine nous a habitués.

\section{IV}

Nous aimerions, pour conclure, présenter quelques éléments de réflexion sur cette rupture saisissante que nous constatons entre Balzac et Flaubert. On peut s'interroger sur les raisons historiques, épistémologiques et aussi générationnelles qui font que vers la deuxième moitié du XIX ${ }^{\mathrm{e}}$ siècle, la ville perd sa fonction symbolique de représentation d'une vérité (politique, personnelle ou utopique) pour devenir une figure du mensonge et de l'illusion, peuplée de héros pitoyables. Quel rôle joue par

\footnotetext{
${ }^{36}$ Ibid., p. 214.

${ }^{37}$ Ibid., p. 468.
} 
exemple la désillusion politique engendrée par l'issue de la révolution de 1848 dans la perception de la ville comme vecteur de trahison et de mensonge? Et en quoi la réécriture urbaine par Haussmann expliquerait-elle que la topographie urbaine se transforme en texte trompeur?

Commençons par le rôle de l'haussmannisation de la ville. À ce propos, il faut rappeler que $L$ 'Éducation sentimentale se situe avant les grands aménagements par Haussmann et évoque donc une ville qui par la suite a connu des transformations et des destructions importantes. L'auteur du roman fait surgir une vision d'un Paris disparu, la conjuration d'une ville-fantôme qui n'est plus, une fantasmagorie d'une époque révolue. Le phénomène de l'haussmannisation n'est guère anecdotique. La réécriture du texte urbain constitue une figure imaginaire, sociale et narrative dont l'impact peut difficilement être surestimé. Comme l'affirme Marie-Claire Banquart, « l'espace parisien de L'Éducation sentimentale se présente d'emblée comme un espace romanesque, mettant en jeu les puissances de l'imaginaire, parce que le lecteur de 1869 le ressent à la fois comme connu et comme disparu ${ }^{38} \gg$. Sans effacer complètement le passé, les transformations urbaines du préfet créent un sentiment de dislocation et d'anéantissement important. L'haussmannisation de la capitale terrasse littéralement l'image de la ville éternelle, et la modernisation urbanistique et sociale que les Parisiens ressentent à tous les niveaux remet en question la vision urbaine vibrante et totalisante que nous rencontrons dans les textes de Balzac ou d'Hugo.

Quant au rôle de la révolution de 1848, on peut s'interroger sur le positionnement politique de l'auteur de L'Éducation sentimentale. Dans l'histoire littéraire, l'image du rentier machiste et réactionnaire colle à la peau d'un Flaubert qui, il faut le dire, a abondamment donné matière dans sa correspondance et dans ses brouillons à la nourrir. La critique acerbe de la bêtise humaine dans laquelle Flaubert excelle prend fréquemment pour cible les idéologies révolutionnaires et leur promesse d'égalité. Persuadé du fait qu'égalité veut surtout dire égalité dans la bêtise, Flaubert ne saurait être considéré comme parangon d'une littérature classiquement engagée. Au grand dam par exemple d'un critique comme Lukács qui, tout en critiquant violemment la prétendue position politique de l'auteur, n'a pu s'empêcher, presque contre son gré, de nous laisser, dans La Théorie du roman, une des plus belles descriptions de la prose flaubertienne ${ }^{39}$. Mais la posture politique flaubertienne qui s'appuie sur la haine de la masse, qui n'est pas la même chose que le mépris du peuple, prend son origine dans ce qui a été pour la génération de Flaubert un des plus grands traumatismes politiques et humains, la révolution de 1848. Celle-ci a vu la Province contester le rôle de guide spirituel revendiqué par Paris, et échouer le rêve révolutionnaire en raison de la trahison du peuple par la bourgeoisie et de l'opportunisme des nantis. L'échec de la révolution, l'étalage généralisé de la bêtise, de la haine et du mensonge politique hanteront Flaubert et fourniront matière à quasiment tous ses romans et à

38 BANQUART, « L'espace urbain de L'Éducation sentimentale », p. 143. Deux remarques de la part du narrateur évoquent la distance qui sépare le lecteur contemporain de l'époque pré-Haussmanienne. L'une est le commentaire sur l'intérieur de Rosanette (« ces élégances, qui seraient aujourd'hui des misères pour les pareils de Rosanette ») et l'autre la réflexion sur « le public des courses, plus spécial dans ce tempslà ». (FLAUBert, L'Éducation sentimentale, p. 172 et p. 266.)

39 Georg LukÁcs, La Théorie du roman, Paris, Éditions Denoël, 1968, p. 125. 
la caractérisation de ses protagonistes. À travers le personnage de Frédéric, antihéros aussi verbeux que velléitaire, il met en évidence le sentimentalisme de cette génération de 1848 qui, enivrée de paroles et de rêves, s'est compromise dans des mensonges politiques et sociaux et a finalement vécu une désillusion cinglante. Cette génération est à mille lieues d'un Rastignac, brûlant de s'approprier la ville et ses promesses. «Je veux faire l'histoire morale des hommes de ma génération; "sentimentale" serait plus vrai. C'est un livre d'amour, de passion; mais de passion telle qu'elle peut exister maintenant, c'est-à-dire inactive ${ }^{40} »$, dit-il dans une lettre à une amie, et le roman nous apprend que «l'action, pour certains hommes, est d'autant plus impraticable que le désir est plus fort ${ }^{41} »$. Cette corrélation inverse entre le désir - démesuré - et la capacité d'action - inexistante - fait de Frédéric le représentant parfait d'une génération qui se perd dans l'assouvissement imaginaire de ses désirs, d'une "génération d'un romantisme attardé, dégradé, qui continue de vivre, sous Louis-Philippe, les révoltes et les rêves nés sous la Restauration; qui verra ses réussites se retourner, et le second Empire naître de la $\mathrm{II}^{\mathrm{e}}$ République $^{42}{ }^{4}$. Dans L'Éducation sentimentale, le mensonge devient alors le moteur d'un récit qui met en scène l'avilissement de l'amour, la dégradation des idéaux politiques, la transformation de l'art en marchandise et la réduction de la langue aux clichés et aux idées reçues ${ }^{43}$. Flaubert rejoint, avec cette vision désillusionnée, un contemporain et son diagnostic non moins sévère de l'époque. Ainsi, dans Le 18 Brumaire de Louis Bonaparte, Karl Marx dresse lui aussi un portrait cinglant de l'époque: "Passions sans vérité, vérités sans passions; héros sans hérö̈sme, histoire sans événements; développement dont la seule force motrice semble être le calendrier, fatiguant par la répétition constante des mêmes tensions et des mêmes détentes ${ }^{44}$. " L'impossibilité de donner un sens à l'histoire, narrative et politique, d'y trouver une vérité objective est certes affligeante d'un point de vue politique. Elle a toutefois, dans L'Éducation sentimentale, donné lieu à une poétique moderne qui tire subtilement parti du retardement de l'assouvissement et où « par un remarquable et mélancolique paradoxe, l'échec devient l'origine de la valeur, la conscience [et le vécu] de ce que la vie a refusé, la source même d'où semble jaillir la plénitude de la vie ${ }^{45} »$. Du mensonge et de l'illusion naît une prose radicalement moderne, ou, pour citer Amédée de Cesena, dans Le Figaro du 20 novembre 1869: « Est-ce bien un roman? C'est peut être le roman de l'avenir, comme la musique de Wagner est la musique de l'avenir, comme la peinture de Courbet est la peinture de l'avenir ${ }^{46}$. »

${ }^{40}$ Gustave Flaubert, Correspondance III, éd. par Jean Bruneau, Paris, Gallimard (coll. «Bibliothèque de la Pléiade »), 1991, p. 409. Lettre à Mademoiselle Leroyer de Chantepie, 6 octobre 1864.

${ }^{41}$ Flaubert, L'Éducation sentimentale, p. 230.

${ }^{42}$ Claudine Gothot-MERSCH, « Introduction à L'Éducation sentimentale », in FLAUBERT, L'Éducation sentimentale, p. 16.

${ }^{43}$ Pour une réflexion sur le statut des idées reçues dans l'œuvre de Flaubert, voir Anne HersCHBERGPIERROT, «Introduction » à Gustave FlaUbert, Le Dictionnaire des Idées reçues et Le Catalogue des idées chic, éd. par Anne Herschberg-PIERrot, Paris, Libraire Générale Française, 1997, p. 5-43.

${ }^{44}$ Karl MarX, Le 18 Brumaire de Louis Bonaparte, Paris, Éditions Mille et une nuits, 1997, p. 53.

45 LukÁcs, La Théorie du roman, p. 125.

46 Amédée de CesenA, « À propos de L'Éducation sentimentale », Le Figaro, 20 novembre 1869. 
